\title{
Metaoptics with Nonrelativistic Matter Waves
}

\author{
T. Taillandier-Loize, ${ }^{1}$ J. Baudon, ${ }^{1}$ M. Hamamda, ${ }^{1}$ G. Dutier, ${ }^{1}$ V. Bocvarski, ${ }^{2}$ \\ M. Boustimi, ${ }^{3}$ F. Perales, ${ }^{1}$ and M. Ducloy ${ }^{1}$ \\ ${ }^{1}$ Laboratoire de Physique des Lasers (CNRS-UMR 7538), Université Paris 13, 99 Av. J.B. Clément, 93430 Villetaneuse, France \\ ${ }^{2}$ Atomic and Molecular Physics Group, Institute of Physics, Belgrade, Pregrevica, 11080 Zemun, Serbia \\ ${ }^{3}$ Physics Department, Umm Al Qura University, P.O. Box 715, Mecca, Saudi Arabia
}

Correspondence should be addressed to J. Baudon, jacques.baudon@univ-paris13.fr

Received 6 June 2012; Revised 22 August 2012; Accepted 24 August 2012

Academic Editor: Pavel A. Belov

Copyright (C) 2012 T. Taillandier-Loize et al. This is an open access article distributed under the Creative Commons Attribution License, which permits unrestricted use, distribution, and reproduction in any medium, provided the original work is properly cited.

The counterpart of metamaterials in light optics for nonrelativistic matter waves governed by the Schrödinger equation can be found by transiently reversing the group velocity using a so called comoving potential. Possible applications to wave-packet dynamics, atom interferometry, and atom deceleration are described.

\section{Introduction}

The genuine concept of "meta" materials for electromagnetic waves originates from the now famous Veselago's paper published in 1967 [1]. The basic idea is that, in a material with negative electric permittivity $(\varepsilon<0)$ and negative magnetic permeability $(\mu<0)$, Maxwell equations impose that the wave vector $\mathbf{k}$ and the Poynting vector $\mathbf{S}$ of a planar wave have opposite directions and, because of causality, the effective optical index is real negative: $n=-(\varepsilon \mu)^{1 / 2}$. The realisation of such artificial or "meta" materials, also called left-handed materials (LHM), in a wide range of wavelengths, has been-and continues to be-the subject of considerable theoretical and experimental efforts [2-4]. Compared to an ordinary material with a positive index, a metamaterial has a similar group velocity, whereas its phase velocity is reversed. This gives rise to the negative refraction phenomenon, owing to which so-called "meta" lenses are conceivable. The concept is rather easily extended to matter waves, provided that the effective mass of the particle be zero or close to zero, as it is the case for electrons in graphene, governed by a (relativistic) Dirac equation [5].

Paradoxically the situation is much more intricate with nonrelativistic particles, as atoms having a thermal velocity (a few hundreds $\mathrm{ms}^{-1}$ ), the dynamics of which is governed by the Schrödinger equation. The first obstacle is the inability of atoms to penetrate dense matter: hence a "material" should be replaced by a "medium", namely, some external potential created in vacuum. A second difficulty comes from the fact that, in this situation, the phase velocity is an ambiguous concept since it is gauge dependent and its inversion appears to be problematic, if not meaningless. Nevertheless the key property of a metamedium lies in the opposite directions of phase and group velocities, a property which will be realised in our case by simply reversing the group velocity. Obviously, given a source of atoms, this property has necessarily a transient character since the group velocity is associated to the density of the probability flux which should finally be oriented outwards from the source. As a consequence, the external potential, assumed to depend on a single spatial coordinate $(x)$, must be also time dependent, of the type $V(x, t)$. In the following, the variation in $x$ of this potential will be considered as being slow at the de Broglie wavelength $(\lambda)$ scale, allowing us to use "short wavelength approximations," for example, WKB or iconal approximation. As shown in part 2, comoving potentials [6], of the general form

$$
V(x, t)=V_{0} s(t) \cos \left(2 \pi \frac{x}{\Lambda}\right)
$$


where $V_{0}$ is a constant amplitude, $s(t)$ is a normalized signal of finite duration, are able to cause the searched inversion of the group velocity and to induce a negative refraction upon the atomic trajectory [7]. The direct observation of this negative refraction on atomic trajectories implies a low velocity and/or a sufficiently high magnitude $V_{0}$, that is, in the case of a magnetic potential, a sufficiently intense magnetic field (typically a few hundreds Gauss at a velocity of a few $\mathrm{ms}^{-1}$. An atom interferometer as a Stern-Gerlach interferometer [8] is a much more sensitive tool to evidence the effect, in so far as it transforms a phase shift into a variation of intensity (part 3).

Two other consequences of the group-velocity inversion are worth to be noted: (i) primarily the negative refraction concerns the motion of a wave packet centre, but it affects also the shape of this wave packet, especially its widthwhich is reduced-, along the same general trend, namely, a transient time reversal [9]; (ii) for similar reasons, the fact that the potential is time dependent results into a nonconservation of energy and more precisely (in the case of negative refraction) into a decrease of the atom velocity. This phenomenon plays an important role in atom interferometry. It can be used to slow down atoms (part 4). As the total length of such a slower is an increasing function of the spatial period $\Lambda$ of the potential, there is a great advantage to make use of a comoving optical potential for which $\Lambda$ is of a few hundreds of nm [10].

Whilst they can give rise to similar effects (together with other specific effects), metamedia for atomic waves are basically different from metamaterials for light optics essentially because of the fundamental difference existing between the related wave equations (Schrödinger versus Maxwell or Dirac). To conclude (part 5), owing to the relative simplicity of their realisation as well as their large domain of applicability, metamedia are expected to play in the future a significant role in atom optics. Nevertheless note that a distinct approach to negative refraction for ultra-cold atoms, based upon "quantum simulators," allows one to simulate condensed matter physics processes with cold atoms (For instance honeycomb optical lattices may be used to reproduce electron dynamics in grapheme [11, 12]. Also specific non-Abelian gauge potentials, simulated with light fields of given wave-vectors and frequencies allow one to assign a quasi-null effective mass to ultracold atoms $(v<1 \mathrm{~cm} / \mathrm{s})$ [13]. Thus, an adequate Klein potential barrier should induce negative refraction.) [11-13]. In the following of this paper we shall not consider this type of situations.

\section{General Principle: Negative Index}

The concept of comoving field of the form given previously, together with its generic property to fashion the momentum $(k)$-dependence of the resulting phase shift, have been introduced in 1997 [6]. Indeed it can be shown [10], using the WKB approximation, that for a field differing from zero within a given interval $\left[0, \tau_{1}\right]$, an incident plane wave $\Psi_{0}(k, x, t)$ of specific momentum $k$, freely propagating along the direction $x$, is altered by the comoving potential via a simple phase factor, becoming $\Psi=\Psi_{0} e^{i \varphi(k, t)}$ with

$$
\begin{aligned}
\varphi(k, t)= & -\hbar^{-1} \int_{0}^{T} d t^{\prime} V_{0} s\left(t^{\prime}\right) \cos \left(2 \pi \frac{\hbar k}{m \Lambda} t^{\prime}\right) \\
& -\hbar^{-1} V_{0} s\left(\tau_{1}^{-}\right) \Theta\left(t-\tau_{1}\right) \cos \left(2 \pi \frac{\hbar k}{m \Lambda} \tau_{1}\right)\left(t-\tau_{1}\right) .
\end{aligned}
$$

In (2), $T=\min \left[t, \tau_{1}\right], \tau_{1}^{-}$is smaller than and arbitrarily close to $\tau_{1}, m$ is the atom mass and $\Theta$ the Heaviside function. The second term in (2) results from the time-dependence of $V$, hence the nonconservation of energy. It warrants the continuity of $\varphi$ and $\Psi$ and their derivatives at $t=\tau_{1}$.

Let us now consider a wave packet, the momentum distribution of which, $\rho(k)$, is centred at $k_{0}$. Using the stationary-phase approximation, it is seen that the potential induces a spatial shift $\delta x_{c}$ upon the motion $x_{c 0}(t)$ of the wave packet centre:

$$
x_{c}=x_{c 0}+\delta x_{c}=\frac{\hbar k_{0}}{m} t-\left[\partial_{k} \varphi\right]_{k_{0}}
$$

which gives:

$$
\begin{aligned}
\delta x_{c}(t)=- & \frac{2 \pi V_{0}}{m \Lambda} \\
\times & {\left[\int_{0}^{T} t^{\prime} s\left(t^{\prime}\right) \sin \left(\frac{2 \pi \hbar k_{0} t^{\prime}}{m \Lambda}\right) d t^{\prime}+\tau_{1} s\left(\tau_{1}\right) \Theta\left(t-\tau_{1}\right)\right.} \\
& \left.\quad \times \sin \left(\frac{2 \pi \hbar k_{0} \tau_{1}}{m \Lambda}\right)\left(t-\tau_{1}\right)\right] .
\end{aligned}
$$

For $t>\tau_{1}$, the integral part takes a finite limiting value, whereas the other term, linear in $\left(t-\tau_{1}\right)$, corresponds to a definite change

$$
\delta v\left(\tau_{1}\right)=-\frac{2 \pi V_{0}}{m \Lambda} \tau_{1} s\left(\tau_{1}\right) \sin \left(\frac{2 \pi \hbar k_{0} \tau_{1}}{m \Lambda}\right)
$$

of the final velocity. This change becomes negligible for comoving pulses of a sufficiently long duration, provided that the product $\tau_{1} s\left(\tau_{1}\right)$ tends to zero when $\tau_{1} \rightarrow \infty$. The important point here is that, by a proper choice of $s(t)$, the $k$-dependence of $\varphi$ can be made such that the group velocity (i.e., the velocity of the wave packet centre) be transiently negative. A trajectory initially in plane $x, z$, with initial velocity components $v_{x 0}>0, v_{z 0}>0$, remains in this plane, exhibiting the negative refraction since $v_{x}$ becomes transiently negative whereas the motion along $z$ remains unaltered. For a sufficiently large value of $\tau_{1}$, the behaviour of the trajectory is similar to that of a ray traversing a negative-index flat plate with parallel surfaces. Figure 1(a) shows an example of such a trajectory for a metastable argon atom $\mathrm{Ar}^{*}\left({ }^{3} \mathrm{P}_{2}\right)$, spin polarized in Zeeman state $M=+2$, experiencing a magnetic comoving potential with $V_{0}=$ $2 g \mu_{B} B_{0}, g$ being the Landé factor, $\mu_{B}$ the Bohr magneton and $B_{0}=50 \mathrm{mT}$ the magnetic field intensity. In this example, 


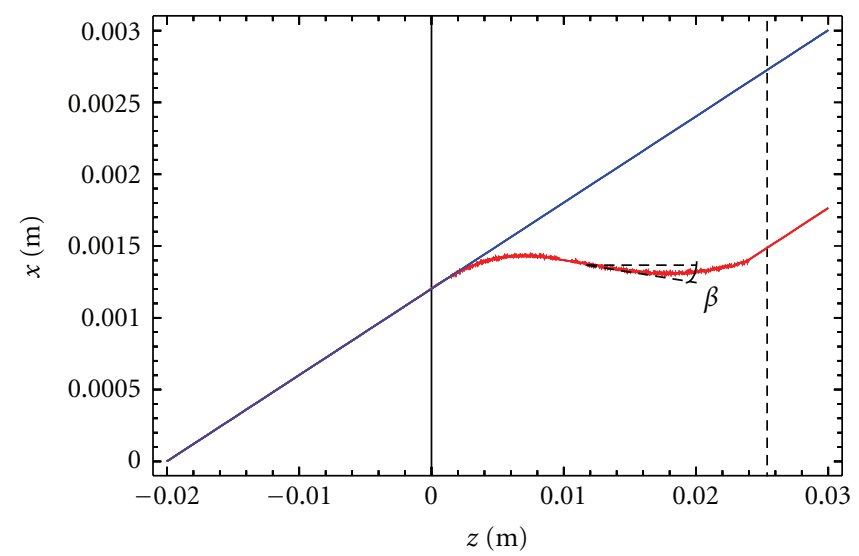

(a)

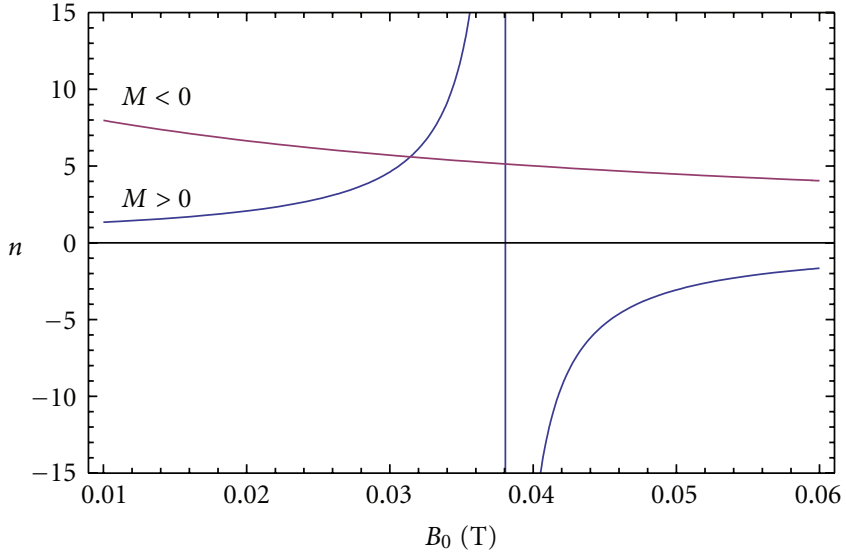

(b)

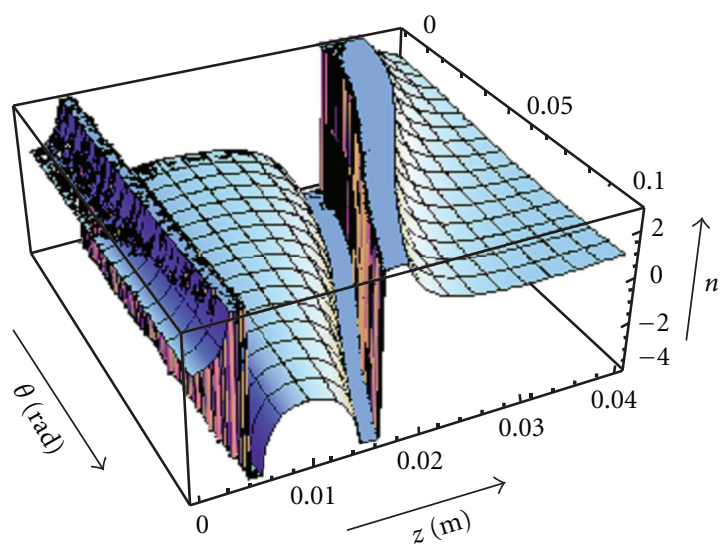

(c)

FIGURE 1: (a) Example of negative refraction of metastable argon atoms $\mathrm{Ar} *\left({ }^{3} \mathrm{P}_{2}\right)$ polarized in Zeeman state $M=+2$. Lower red curve: atomic trajectory in plane $z, x$ under the action of a magnetic field comoving in the $x$ direction. Blue straight line: free propagation. Parameters of the comoving field are as follows (see text): spatial period $\Lambda=5 \mathrm{~mm}$, magnitude of the magnetic field $B_{0}=49 \mathrm{mT}$, velocity components $v_{0 x}=1.2 \mathrm{~ms}^{-1}, v_{0 z}=20 \mathrm{~ms}^{-1}$, time constant $\tau=0.37 \mathrm{~ms}$, duration $\tau_{1}=4.0 \mathrm{~ms}$. The point-like source is on the $z$ axis at $z=-2 \mathrm{~cm}$. The angle $\beta$ between the local velocity and the $z$ axis (see text) is shown. (b) Effective constant index (see text, (6a)) as a function of $B_{0}$. Curve $>0$ : the index is negative for $B_{0}>38 \mathrm{mT}$. Curve $M<0$ : the index (multiplied by 10) is positive for any value of $B_{0}$. (c) Index $n$ as a function of $z$ and $\theta$, derived from an ensemble of trajectories similar to that shown in (a), but with different incidence angles. Positions in the $(z, \theta)$ plane where $n \rightarrow \pm \infty$ correspond to points where the atom velocity is parallel to the $z$ axis. In between these two values, $n$ is negative.

$\Lambda=5 \mathrm{~mm}, v_{x 0}=1.2 \mathrm{~m} \mathrm{~s}^{-1}, v_{z 0}=20 \mathrm{~m} \mathrm{~s}^{-1}$. The timedependent signal is $s(t)=\exp [-t / \tau]$ with $\tau=0.37 \mathrm{~ms}$ and $\tau_{1}=4 \mathrm{~ms}$. Note that, at these low velocities, the lateral shift of the trajectory is rather large $(1.2 \mathrm{~mm})$ in spite of the relatively modest value of the magnetic field. Other values of $M(+1,0,-1,-2)$ would lead to shifts proportional to $M$, which means that the comoving potential acts as an efficient beam splitter. More generally the comoving potential zone behaves as a multirefringent plate.

Assuming that deflection angles are small, by comparison with the light-optics counterpart under similar conditions, one can obtain an effective index $n$ given, for $\tau_{1} \gg \tau$, by [14]

$$
n^{-1} \approx 1-\frac{2 \tau^{3} M g \mu_{B} B_{0}}{m \tau_{\max }}\left(\frac{2 \pi}{\Lambda}\right)^{2}
$$

where $\tau_{\max }$ is an estimate of the time at which $\delta x_{c}$ reaches its asymptotic value $\left(\tau_{\max } \approx 1.6 \tau\right)$. As it is seen in Figure $1(\mathrm{~b})$, for $M>0$, the effective index becomes negative for values of $B_{0}$ larger than a critical value, $B_{c}=38 \mathrm{mT}$ in the present case. The fact that $n \rightarrow \pm \infty$ at $B_{0}=B_{c}$ is not really a singularity since it simply means, in the light-optics analogy, that the ray inside the plate is normal to the plate's surfaces. On the other hand, for $M<0, n$ is positive for any value of $B_{0}$, giving rise to ordinary positive refraction. The effective index given by (6a) is a constant related to a simplified trajectory consisting of three portions of straight lines. In the case of a comoving field, on the other hand, the index is $z$ dependent. It can be derived from the usual ray equation in an inhomogeneous medium: $\left(n \mathbf{r}^{\prime}\right)^{\prime}=\nabla n$, where $\left(^{\prime}\right)=d / d s, s$ being the curvilinear abscissa. As a function of the variable 
$X(z)=(d x / d z)$, the element of curvilinear abscissa is $d s=$ $d z \sqrt{1+X^{2}}$. Then the index is simply:

$$
n=A \frac{\sqrt{X^{2}+1}}{X}
$$

where $A$ is a constant such that $n(z=0)=1$. This expression of $\mathrm{n}$ simply reflects the Snell-Descartes refraction law in a medium stratified by planes orthogonal to $z$, namely

$$
n \sin \beta=\text { constant, }
$$

where $\beta=\operatorname{ArcTan}\left(v_{x} / v_{0 z}\right)$. Finally, once modelled the trajectory, the index profile $n(z)$ can be derived. Note that $n$ has the sign of $X$ and, as expected, it is infinite when $X=0$. Actually especially at large values of $z$, the index profile derived from $((6 b)-(6 c))$ depends on the angle of incidence $\theta$ since the constant in $(6 \mathrm{c})$ is $\sin \theta$. On the other hand the medium is invariant in any translation along $x$. As a conclusion the metamedium is anisotropic. Figure 1(c) shows the $2 \mathrm{D}$ profile of the index $n(z, \theta)$ derived from an ensemble of trajectories similar to that of Figure 1(a), but calculated with different incidence angles ranging from 0 to $0.1 \mathrm{rad}$.

\section{Negative-Index Medium in a Stern-Gerlach Atom Interferometer}

A standard Stern-Gerlach atom interferometer [8], also called some years later "spin-echo experiment," in analogy to the well-known method of neutron spin-echo $[15,16]$, is a longitudinal polarisation interferometer in which an integrable static magnetic field profile $B(x)$, that is, a $M$ dependent magnetic potential $W(x)=g \mu_{B} M B(x)$, induces upon a planar wave (of momentum $k$ ) describing the external motion along $x$, a phase shift of the form $M \phi$. In the following semiclassical approximation:

$$
\phi \approx \frac{1}{\hbar v} \int_{-\infty}^{+\infty} W(x) d x
$$

where $v \approx \hbar k / \mathrm{m}$. Starting from a given Zeeman state $\left|M_{0}\right\rangle$ issued from a polarizer, for example, a Stern-Gerlach polarizer, one first prepares, using Majorana transitions (fast rotation of a tiny magnetic field) [17], a linear superposition of $M$-states

$$
\left|\Psi_{i}\right\rangle=\sum_{M} a_{M_{0} M}|M\rangle
$$

where the $a_{M_{0} M}$ are constant coefficients. Beyond the field profile $B(x)$, it becomes

$$
\left|\Psi_{f}\right\rangle=\sum_{M} a_{M_{0} M} e^{i M \phi}|M\rangle
$$

Then a second Majorana zone generates the new combination (where the $b_{M M^{\prime}}-\mathrm{s}$ are constant)

$$
\left|\Psi_{o}\right\rangle=\sum_{M, M^{\prime}} a_{M_{0} M} b_{M M^{\prime}} e^{i M \phi}\left|M^{\prime}\right\rangle .
$$

Finally, an analyzer (similar to the polarizer) selects a specific Zeeman state $\left|M_{1}\right\rangle$ and one measures the final intensity

$$
I_{1}=\left|\sum_{M} a_{M_{0} M} b_{M M_{1}} e^{i M \phi}\right|^{2} .
$$

It contains interference terms in $\phi$ which can be evidenced by varying the magnitude of the magnetic field or the velocity. In place of a static field profile, a comoving field can be used as well, as it has been demonstrated in [6] with a beam of fast $\left(v=10^{4} \mathrm{~ms}^{-1}\right)$ metastable hydrogen atoms $H^{*}\left(2^{2} S_{1 / 2}, F=1\right)$. Very recently, experiments dealing with similar questions have been realized by Sulyok et al. with a beam of neutrons at a velocity of $2000 \mathrm{~ms}^{-1}$, in a so-called perfect crystal interferometer [18]. The magnetic potential they use is a sum of terms of the form $C(x) V_{k} \cos \left(\omega_{k} t+\right.$ $\left.\varphi_{k}\right)$, where $C(x)$ is a square function of a definite width $L$ and $V_{k}, \omega_{k}, \varphi_{k}$ are constants. It might seem different from our comoving potential. However it can be readily seen (by taking the Fourier transform of the spatial dependence) that this potential is actually a sum of comoving terms.

The main questions that arise about the use of comoving potentials as phase objects in an interferometer deal with similarities and differences they present with respect to static potentials. The first specificity of comoving potentials is that, because of the transient character of the effect, a treatment using wave packets is needed. Apart from the narrowing effect mentioned previously (difficult to observe except at low velocity), the first consequence of that is the critical velocity dependence of the interference effect, particularly when a purely sinusoidal signal of the type $s(t)=\cos (2 \pi \nu t)$ is used. Indeed in that case there exists a "resonant" atomic velocity coinciding with the field velocity $u_{\text {com }}=v \Lambda$. This resonant velocity can correspond to a bright fringe or a dark fringe, according to the value of the magnitude $B_{0}$ of the magnetic field (which is generally low, less than $100 \mathrm{mG}$ ). This central fringe is surrounded by few other fringes within the envelope of the resonance. This phenomenon has been observed using a time-of-flight technique, with a single zone (see Figure 2, taken from [6]) or a double zone of comoving field.

Another manifestation, specific of comoving potentials, appears when a nonsinusoidal signal of a finite duration is used, for example, $s(t)=e^{-t / \tau}$ for $0<t<\tau_{1}$, $=0$ elsewhere. When the value of the cut-off time $\tau_{1}$ is large compared to $\tau$, final velocities $v_{+}$and $v_{-}$related to sublevels $M>0$ and $M<0$, are almost equal to the initial velocity $v_{0}$. On the contrary, when $\tau_{1}$ is comparable to or smaller than $\tau$, one gets $v_{+}<v_{0}$ (beginning of a negative refraction) and $v_{-}>v_{0}$. At $\tau=\tau_{1}$, the abscissas of the "+" and "-" wave packets are such that $x_{+}<x_{-}$, then their mutual longitudinal separation $\Delta_{L} x$ monotonously increases with $t$ or $x$, which cancels any interference effect between them (once remixed), in other words a total loss of contrast as the distance to the detector is increased. Figure 3(a) shows $\Delta_{L} x$ as a function of $x$ in the realistic case of metastable argon atoms of initial velocity $v_{0}=560 \mathrm{~m} \mathrm{~s}^{-1}$. Whilst the magnitude of $\Delta_{L} x$ may seem small (a fraction of $1 \mu \mathrm{m}$ ), it is much larger than the wavelength $(0.02 \mathrm{~nm})$ and the effect on the interference is drastic. 


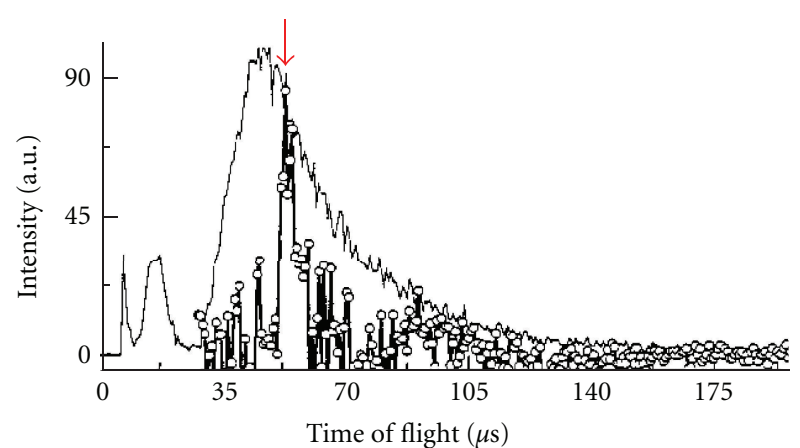

FIgUre 2: Time-of-flight spectrum over a distance of $49 \mathrm{~cm}$, of hydrogen metastable atoms $\left(H^{*} 2 s, F=1\right)$ going out of a Stern-Gerlach atom interferometer (see text). The atom velocity is $10 \mathrm{~km} \mathrm{~s}^{-1}$. Open circles: a comoving field is used as a phase object. The value $B_{0}=6.5 \mu \mathrm{T}$ is chosen to get a central bright fringe. The spatial period is $\Lambda=2 \mathrm{~cm}$ and a unique frequency $v=440 \mathrm{kHz}$ is used. The field velocity is $\Lambda \nu=8.8 \mathrm{~km} \mathrm{~s}^{-1}$, which corresponds to the time of flight indicated by a vertical (red) arrow. Light line: time-of-flight spectrum without the interferometer.

A similar phenomenon is described in [18] for the case of neutron interferometry. In principle it is possible to recover the contrast via the action of a second reversed comoving pulse provided that the characteristics of this second pulse (especially its duration $\tau_{1}$ ) are adjusted such that the final velocities are exactly equal to each other. Figure $3(\mathrm{~b})$ shows an example of such compensation - over a distance of several centimetres-by means of a second reversed pulse similar to the first one but applied $2 \mathrm{~ms}$ later.

Figure 4 shows the high sensitivity of the interferometer operating with metastable argon atoms at thermal velocity $\left(560 \mathrm{~ms}^{-1}\right)$. At such large velocity the inelastic effect induced by the potential pulse is small enough to make the contrast practically independent of the distance at which the detector is placed (from 0.1 to $1 \mathrm{~m}$ ). On the other hand the contrast is reduced at "large" field magnitude, of the order of $0.1 \mathrm{mT}$ or more, because of the increasing spatial separation between the two interfering wave packets and the related decrease of their overlap.

\section{Atom (or Molecule) Slower}

As explained in part 2, the primary effect of a potential pulse, comoving in the $x$ direction, the sign of which is such that (for a sufficient magnitude) it results into a negative refraction, is to reduce the velocity component along $x$ by an amount, derived from (3) (for $t \leq \tau_{1}$ ):

$$
\delta v(t)=\partial_{t} \delta x_{c}=-\frac{2 \pi V_{0}}{m \Lambda} t s(t) \sin \left(\frac{2 \pi \hbar k_{0} t}{m \Lambda}\right) .
$$

In principle this effect can be used to slow down atoms or molecules. For neutrons this was shown to work [19]. However when the initial velocities are in the thermal range (e.g., $560 \mathrm{~ms}^{-1}$ for $\mathrm{Ar}^{*}$ atoms) the predicted reduction of $v$ is quite small, typically of a few $\mathrm{mm} \mathrm{s}^{-1}$, at least (in the case of a magnetic potential) for reasonable values of $B_{0}$, namely less than $0.1 \mathrm{~T}$. As a consequence, to reduce the velocity down

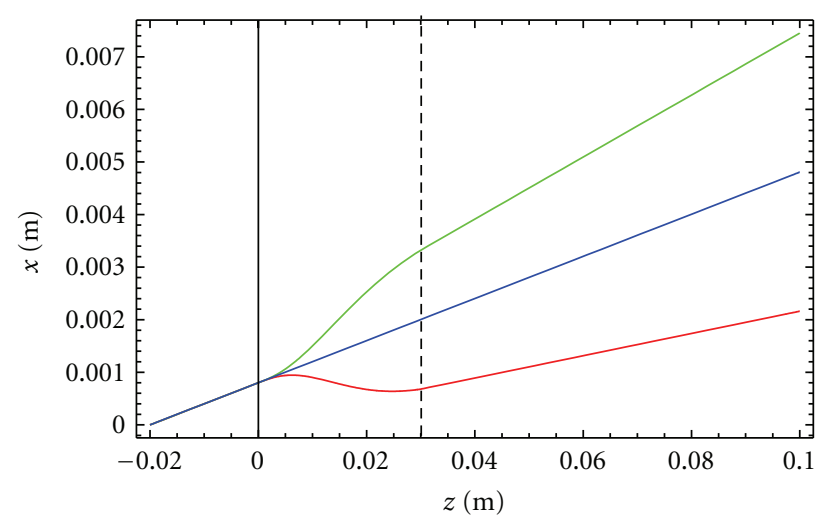

(a)

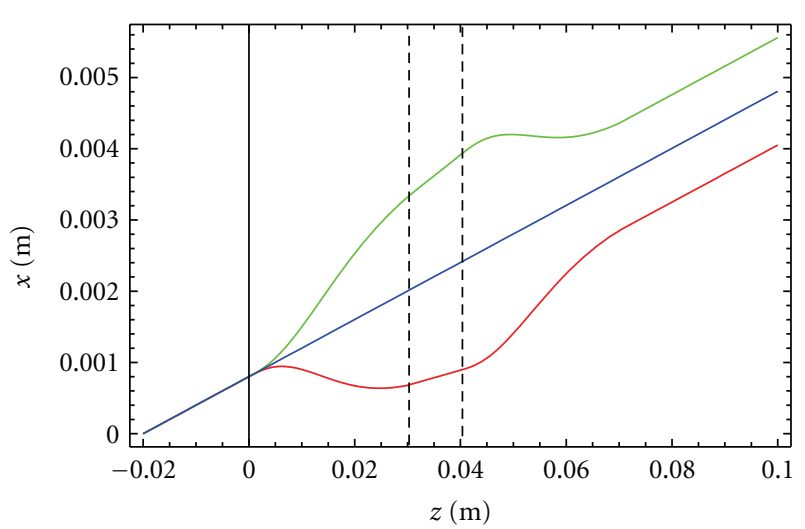

(b)

Figure 3: (a) Effect of a short comoving pulse on the final velocities. The conditions are the same as in Figure 1(a) except the pulse duration, $\tau_{1}=1.5 \mathrm{~ms}$ (instead of $4 \mathrm{~ms}$ ), indicated (converted in $z$ value) by the broken vertical line. It is seen that the final velocity for atoms in state $M=-2$ (upper green trajectory) is larger than for atoms in state $M=+2$ (lower red trajectory). The two related wave packets fligh apart from each other, cancelling the interference effect between them once they are remixed (see text). (b) Compensation of this effect by means of a second reversed comoving pulse. The first pulse is the same as in 3(a) (starting time $t=0$, duration $\left.\tau_{1}=1.5 \mathrm{~ms}, B_{0}=49 \mathrm{mT}\right)$. The second pulse starts at $t=2.0 \mathrm{~ms}$ (second vertical broken line), its duration is also $1.5 \mathrm{~ms}$ and its amplitude is $B_{0}=49 \mathrm{mT}$. Resulting trajectories are shown as in (a). Final velocities become equal to the initial velocity.

to almost zero, a large number of successive pulses is needed. This is made possible by the fact that, immediately after the end of a pulse of duration $\tau_{1}$ the velocity is practically equal to the reduced velocity obtained at time $\tau_{1}$. Then, when (after a short blank) the next pulse is applied, this latter velocity becomes the initial velocity, which is in turn reduced, and so forth. The best choice for the pulse duration $\tau_{1}$ is such that $\left|\delta v\left(\tau_{1}\right)\right|$ derived from (5) takes its first maximum value. For a signal of the form $s(t)=\exp (-t / \tau)$ [6], there exists an optimum value of the time constant $\tau$ leading to an absolute maximum of $\left|\delta v\left(\tau_{1}\right)\right|$. For $v=20 \mathrm{~ms}^{-1}$, the best values are obtained for $\tau=63.3 \mu \mathrm{s}$ and $\tau_{1}=62.6 \mu \mathrm{s}$. Actually they are almost equal to each other and their dependence on $v$ is such that the product of the optimum value of $\tau_{1}$ by $v$ is a constant. 


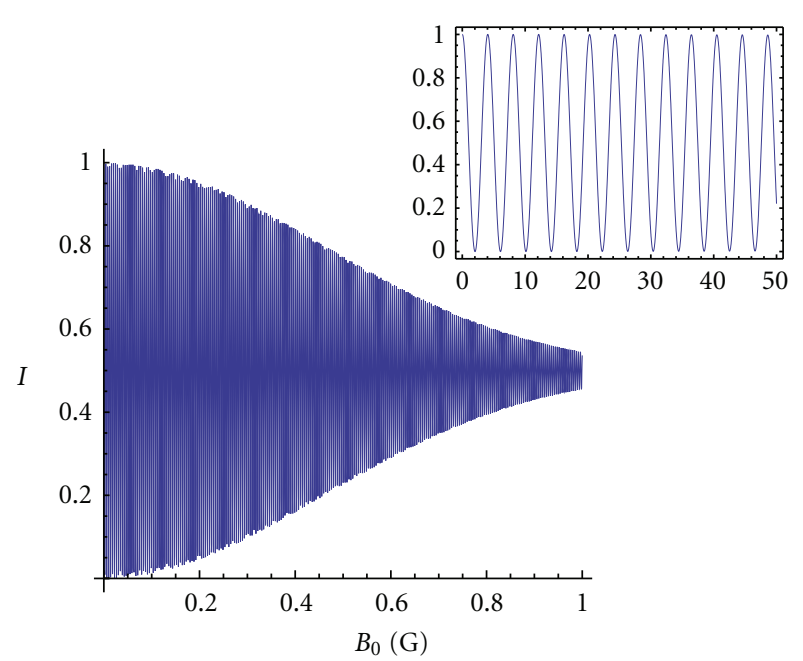

FIgURE 4: Calculated interference pattern of $\operatorname{Ar} *\left({ }^{3} \mathrm{P}_{2}\right)$ atoms passing through a Stern-Gerlach interferometer at $560 \mathrm{~ms}^{-1}$. The phase object is a comoving potential pulse similar to that considered in Figure 1(a), except for the magnitude $B_{0}$ of the field which is now the variable parameter. About 260 fringes are present within the interval $[0,0.1 \mathrm{mT}=1$ Gauss] (see the inset for a zoom over an interval of $5 \mu \mathrm{T}=50$ milliGauss). The contrast decreases together with the overlap of the interfering wave packets (see text).

This means that the atomic path covered through successive pulses is almost a constant.

The present method bears some similarities with the socalled "adiabatic slowing" [20, 21]. This latter method has been applied to a wide variety of species, such as hydrogen atoms, polar and non polar molecules [22], Rydberg atoms and Rydberg molecules [23-25]. Low final velocities (a few $10 \mathrm{~ms}^{-1}$ ) are accessible, but at the price of rather strong fields (e.g., $B_{0}=5.2 \mathrm{~T}$ in $[23-25]$ ). Here, the nature of the force is quite different, since it derives from a special potential depending on both space and time. In principle the method is applicable to the same species, with the advantage that it uses much lower fields.

As shown in [10] an atom slower using magnetic potential pulses ( $B_{0}=80 \mathrm{mT}, \Lambda=5 \mathrm{~mm}$ ) is able to reduce the velocity of metastable argon atoms from $560 \mathrm{~m} \mathrm{~s}^{-1}$ down to almost zero over a distance of $2.2 \mathrm{~m}$, comparable to the total length of a standard Zeeman slower [26]. For a given magnitude of the potential, the length is governed by the spatial period $\Lambda$. In a simple magnetic version, it is almost impossible to reduce it below $1 \mathrm{~mm}$, whereas the use of a dipolar optical potential obtained in a off-resonance standing wave provides us with a huge reduction of the period, which is half the optical wavelength $\Lambda=\lambda_{\text {opt }} / 2(\approx 0.4 \mu \mathrm{m}$ in the case of metastable argon atoms). Far from resonance the general form of such a potential is [27]:

$$
V(x, t) \approx \frac{\hbar \Omega^{2}}{\delta \omega} s(t) \cos ^{2}\left(2 \pi \frac{x}{\lambda_{\mathrm{opt}}}\right),
$$

where $\Omega$ is the Rabi frequency and $\delta \omega$ the detuning. To operate "far from resonance" (to avoid any spontaneous emission), the difference between the laser frequency and the

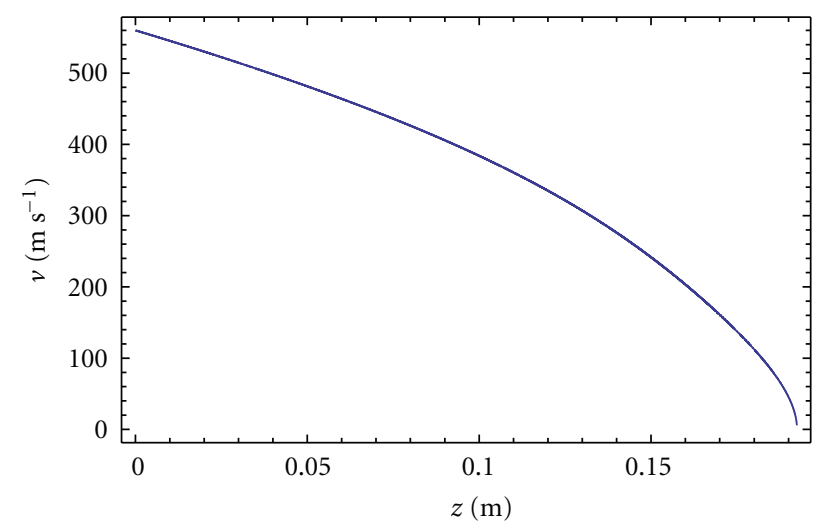

Figure 5: Calculated evolution of the velocity $v$ along the slowing process using optical comoving pulses (see text), as a function of the abscissa $z$. An almost complete stopping is achieved at $z=19 \mathrm{~cm}$.

two resonances appearing in the standing wave, Dopplershifted by $\Delta \omega= \pm k_{\mathrm{opt}} v$, must be large compared to the power-broadened line width $\gamma^{\prime}=(1+\Sigma)^{1 / 2} \gamma$, where $\gamma$ is the natural line width and $\Sigma$ the saturation parameter. To get a magnitude of the potential sufficiently high to achieve the complete slowing over a distance shorter than say, $20 \mathrm{~cm}$, using a reasonable laser power (e.g., $32 \mathrm{~mW} \mathrm{~mm}^{-2}$ ), a moderately large (negative) detuning should be chosen, such as $\delta \omega=-2 \pi 3.45 \times 10^{9} \mathrm{rad} / \mathrm{s}(3.45 \mathrm{GHz})$. This leads to a ratio $=\delta \omega / \gamma^{\prime}=12.49$, large compared to 1 . As the velocity $v$ is lowered, $\Delta \omega$ decreases, tending to zero as $v \rightarrow 0$. Then either the detuning is kept constant and the condition $R \gg 1$ is better and better verified, or $\delta \omega$ is kept equal to $5 \Delta \omega(v)$, allowing us to reduce the intensity (as $v$ ) as well as $\gamma^{\prime}$ (as $v^{1 / 2}$ ), but then the ratio $\mathrm{R}$ decreases as $v^{1 / 2}$, which implies a lower limit for $v(R=1$ at $v=3.59 \mathrm{~m} / \mathrm{s})$. As before a series of many pulses separated from each other by small blanks is applied, each of them (numbered $n$ ) providing a small decrease $|\delta v|$ of the velocity (a few $\mathrm{mm} / \mathrm{s}$ ). The duration $\tau_{1}(n)$ of each pulse is adjusted in such a way that the first maximum value of $|\delta v|$ is reached at the end of the pulse. As previously the path $v \tau_{1}$ covered by the atom during successive pulses is roughly a constant $(\approx 0.12 \mu \mathrm{m})$. Figure 5 shows how the velocity $v$ decreases down to almost zero (with the restriction mentioned before) as a function of $z$. The total number of applied pulses is large (about $210^{6}$ ) but the total length is now $19.2 \mathrm{~cm}$. Note that the method does not imply any permanent magnetic moment of the atom and is applicable for instance to $\mathrm{Ar} *\left({ }^{3} \mathrm{P}_{0}\right)$ metastable atoms.

In addition to the advantages of its short length and the absence of any random spreading of the velocity (at least if the spontaneous emission is negligible), the present decelerator is interesting from the point of view of the atomic density in the phase space. Indeed an important characteristic of comoving fields is their effect on the longitudinal spatial width of the atomic wave-packet. As mentioned previously [9], comoving potentials are able to transiently narrow wave packets, compensating for the freepropagation natural spreading. In the present case this effect 
is very small at the beginning of the deceleration process but becomes more and more important as the velocity decreases. As a result the wave packet width $\delta x$ progressively deviates from the free-propagation width $\delta x_{0}(t)$ to rejoin its initial value $\delta x_{0}(0)$. On the other hand it can be verified, using the Wigner function, that the width $\delta k$ of the momentum distribution remains unchanged, the reason being that the effect of the potential is a pure real phase shift. Consequently the density $(\delta k \delta x)^{-1}$ in the phase space $\left(x, k_{x}\right)$, instead of continuously decreasing, recovers its initial value at the end of the slowing process.

\section{Conclusion}

In this paper comoving fields have been introduced in view of realizing negative-index media for matter waves in the nonrelativistic regime. Because of the fundamental difference between Schrödinger and Maxwell (or Dirac) equations, especially for what concerns the phase velocity, a method quite different from those used in light optics or ultrarelativistic particle optics is needed. The aim of our method is the transient inversion of the group velocity. Phenomena similar to those observed in metamaterials, as the negative refraction, metalens, etc.) are expected. Other properties are specific of our "metamedia." In particular, the evolution of the wave-packet spatial width exhibits unusual features, as a transient narrowing, accompanying the negative refraction and related to time reversal, and also a velocity change in the case of short comoving potential pulses because of the nonconservation of energy.

All these effects, on atom trajectories or wave-packet width evolution, are directly observable provided that the atomic velocities are low, typically of a few $\mathrm{ms}^{-1}$. At higher velocity, like a few hundreds of $\mathrm{ms}^{-1}$, more sensitive techniques are necessary. Atom interferometers in general and Stern-Gerlach interferometers in particular, offer such sensitivity. We have shown that observable optical-index effects appear with magnetic fields as small as a few $\mu \mathrm{T}$.

We have proposed an approach to atom beam deceleration based on dispersive optical forces. Atom stopping should be almost achieved on short distances using a moderate laser power, for example, less than $50 \mathrm{~mW} / \mathrm{mm}^{2}$. The absence of spontaneous emission processes should allow preservation of the transverse coherence properties of the initial beam. The technique is especially applicable to narrow supersonic beams, like metastable rare-gas atom beams, and it is able to provide us with ultra-low-velocity beams for coherent atom optics and atomic interferometry. It is also a promising technique applicable to slowing down not solely diamagnetic atoms (such as metastable argon atoms in the ${ }^{3} \mathrm{P}_{0}$ state) but also molecules since any optical pumping toward molecular levels other than those interacting with light is absent. Slowing and trapping of molecules is a subject of a particular importance in the investigation of cold collisions (determination of intermolecular potentials at large distances, resonances of various kinds).

\section{Acknowledgments}

The authors from Laboratoire de Physique des Lasers acknowledge the Institut Francilien de Recherche sur les Atomes Froids (IFRAF) for support.

\section{References}

[1] V. G. Veselago, "The electrodynamics of substances with simultaneously negative values of epsilon and $\mu$," Soviet Physics Uspekhi, vol. 10, no. 4, pp. 509-514, 1968.

[2] J. Pendry, "Negative refraction makes a perfect lens," Physical Review Letters, vol. 85, no. 18, pp. 3966-3969, 2000.

[3] C. Foteinopoulou, E. N. Economou, and C. M. Soukoulis, "Refraction in media with a negative refractive index," Physical Review Letters, vol. 90, Article ID 107401, 4 pages, 2003.

[4] A. Sambale and D.-G. Welsch, "van der Waals interaction and spontaneous decay of an excited atom in a superlens-type geometry ," Physical Review A, vol. 78, Article ID 053828, 10 pages, 2008.

[5] V. V. Klimov, J. Baudon, and M. Ducloy, "Comparative focusing of Maxwell and Dirac fields by negative-refraction half-space," Europhysics Letters, vol. 94, no. 2, Article ID 20006, 2011.

[6] R. Mathevet, K. Brodsky, B. J. Lawson-Daku, C. Miniatura, J. Robert, and J. Baudon, "Atomic interferences in a comoving magnetic field," Physical Review A, vol. 56, no. 4, pp. 29542958, 1997.

[7] J. Baudon, M. Hamamda, J. Grucker et al., "Negative-index media for matter-wave optics," Physical Review Letters, vol. 102, no. 14, Article ID 140403, 2009.

[8] C. Miniatura, J. Robert, O. Gorceix et al., "Atomic interferences and the topological phase," Physical Review Letters, vol. 69, no. 2, pp. 261-264, 1992.

[9] M. Hamamda, F. Perales, G. Dutier et al., "Time reversal in matter-wave optics," European Physical Journal D, vol. 61, no. 2, pp. 321-325, 2011.

[10] M. Hamamda, M. Boustimi, F. Correia et al., "Atom slowing via dispersive optical interactions," Physical Review A, vol. 85, no. 2, Article ID 023417, 5 pages, 2012.

[11] S. L. Zhu, B. Wang, and L. M. Duan, "Simulation and detection of Dirac fermions with cold atoms in an optical lattice," Physical Review Letters, vol. 98, no. 26, Article ID 260402, 4 pages, 2007.

[12] K. L. Lee, B. Grémaud, R. Han, B. G. Englert, and C. Miniatura, "Ultracold fermions in a graphene-type optical lattice," Physical Review A, vol. 80, no. 4, Article ID 043411, 2009.

[13] G. Juzeliunas, J. Ruseckas, M. Lindberg, L. Santos, and P. Öhberg, "Quasirelativistic behavior of cold atoms in light fields," Physical Review A, vol. 77, no. 1, Article ID 011802, 4 pages, 2008.

[14] M. Hamamda, PhD thesis, University Paris 13.

[15] F. Mezei, "Neutron spin echo: a new concept in polarized thermal neutron techniques," Physik, vol. 255, no. 2, pp. 146160, 1972.

[16] M. De Kieviet, D. Dubbers, Ch. Schmidt, D. Scholz, and U. Spinola, "3 He spin echo: new atomic beam technique for probing phenomena in the nev range," Physical Review Letters, vol. 75, no. 10, pp. 1919-1922, 1995.

[17] E. Majorana, "Atomi orientati in campo magnetico variabile," Nuovo Cimento, vol. 9, no. 2, pp. 43-50, 1932. 
[18] G. Sulyok, H. Lemmel, and H. Rauch, "Neutrons in a timedependent magnetic field: photon exchange and decoherence modeling," Physical Review A, vol. 85, no. 3, Article ID 033624, 7 pages, 2012.

[19] L. Niel and H. Rauch, "Acceleration, deceleration and monochromatization of neutrons in time dependent magnetic fields," Zeitschrift für Physik B, vol. 74, no. 1, pp. 133-139, 1989.

[20] H. L. Bethlem, F. M. H. Crompvoets, R. T. Jongma, S. Y. T. Van de Meerakker, and G. Meijer, "Deceleration and trapping of ammonia using time-varying electric fields," Physical Review A, vol. 65, no. 5, Article ID 053416, 20 pages, 2002.

[21] H. L. Bethlem, G. Berden, and G. Meijer, "Decelerating neutral dipolar molecules," Physical Review Letters, vol. 83, no. 8, pp. 1558-1561, 1999.

[22] E. Narevicius, A. Libson, C. G. Parthey et al., "Stopping supersonic beams with a series of pulsed electromagnetic coils: an atomic coilgun," Physical Review Letters, vol. 100, no. 9, Article ID 093003, 2008.

[23] Y. Yamakita, S. R. Procter, A. L. Goodgame, T. P. Softley, and F. Merkt, "Deflection and deceleration of hydrogen Rydberg molecules in inhomogeneous electric fields," Journal of Chemical Physics, vol. 121, no. 3, p. 1419, 2004.

[24] E. Vliegen, H. J. Worner, T. P. Softley, and F. Merkt, "Nonhydrogenic effects in the deceleration of Rydberg atoms in inhomogeneous electric fields," Physical Review Letters, vol. 92, no. 3, Article ID 033005, 4 pages, 2004.

[25] N. Vanhaecke, D. Comparat, and P. Pillet, "Rydberg decelerator using a travelling electric-field gradient," Journal of Physics B, vol. 38, no. 2, p. S409, 2005.

[26] W. D. Phillips and H. J. Metcalf, "Laser deceleration of an atomic beam," Physical Review Letters, vol. 48, no. 9, pp. 596599, 1982.

[27] H. J. Metcalf and P. van der Straten, Optical Cooling and Trapping, Springer, New York, NY, USA, 2001. 

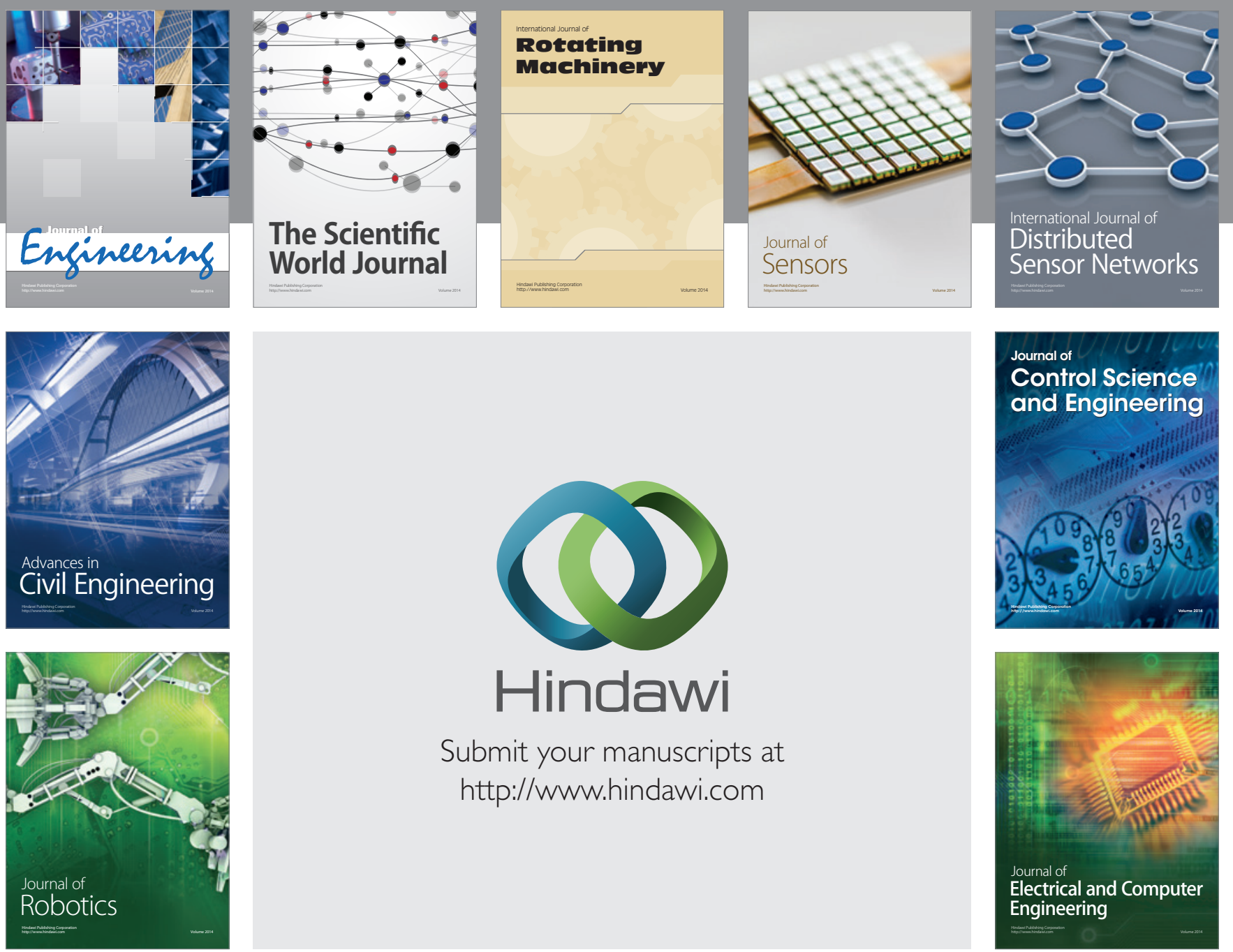

Submit your manuscripts at

http://www.hindawi.com
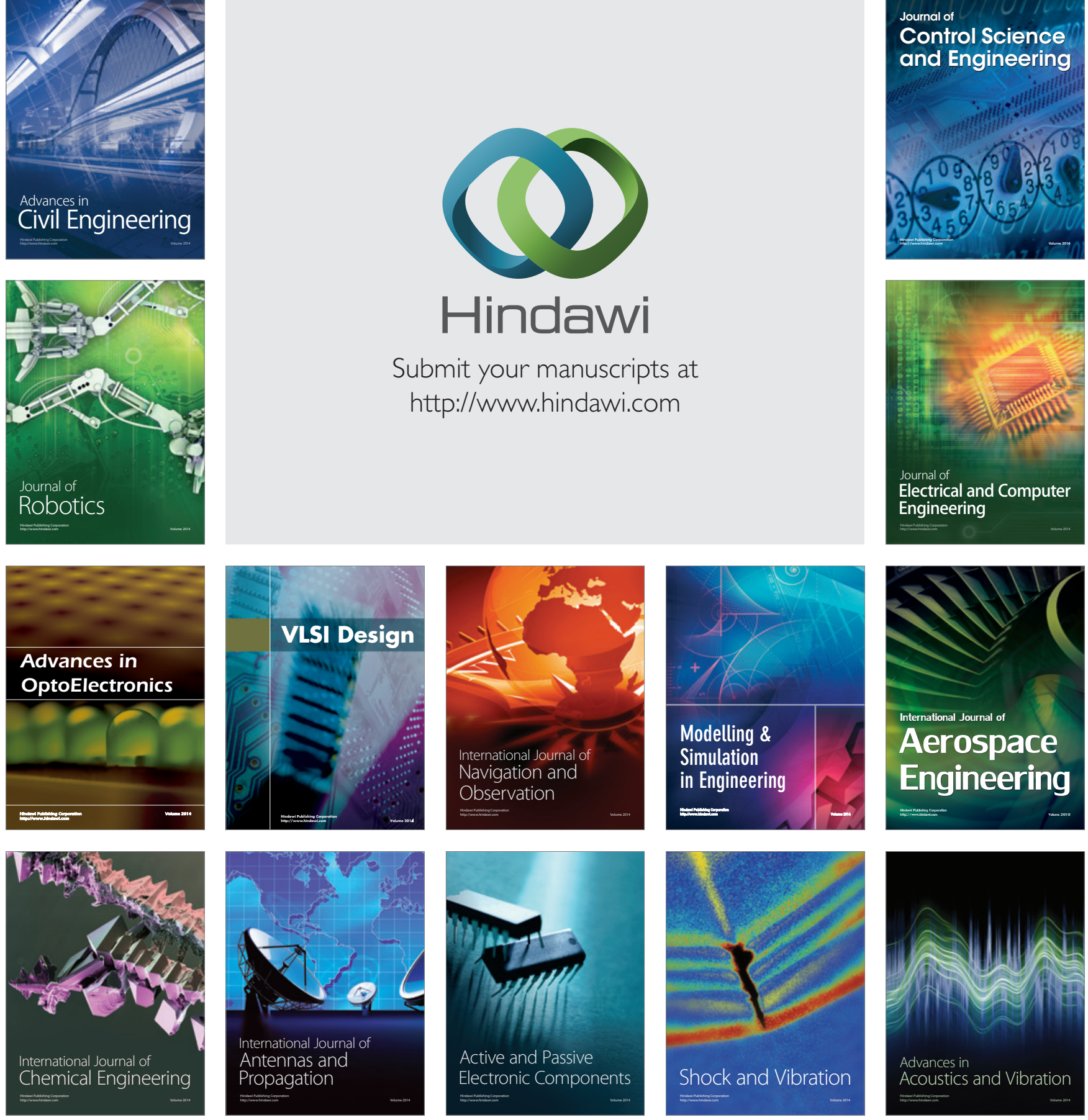